\title{
The expression of an infinite lower semi-matrix in terms of its idempotent and nilpotent elements
}

\author{
By C. E. Gurr. \\ Communicated by Professor H. W. Turnbull. \\ (Received 15th October, 1938. Read 4th November, 1938.) \\ (Received in revised form 6th February, 1939.)
}

The importance of matric algebras in Function Theory and in Physics (Birtwistle-The new Quantum Mechanics; and Courant and Hilbert-Methoden der mathematischen Physik) has resulted in comprehensive works on finite matrices ${ }^{1}$. Very little progress has, however, been made in the necessary algebras of infinite matrices.

Some general theory, together with application to Function Theory and Physics, has been devcloped by Dienes ${ }^{2}$ and supplemented $^{3}$ by recent papers by $R$. Cooke. Little is yet known of functional properties, and before the algebra of infinite matrices can be fully developed it is essential that knowledge should be gained of the structural properties of the matrix. This work is being pursued at Birkbeck College by a group of research students working under Dr Dienes. One of the problems he put forward at a lecture to this group was the examination of the structure of infinite semi-matrices, suggesting, for distinct roots, the form $A_{i} / C_{i}$ (as given in the paper) for the idempotent element. Under his guidance I have been able to obtain the structural form of infinite semi-matrices of a certain class on a parallel with the exposition ${ }^{4}$ given by Wedderburn for finite matrices.

Direct examination of the matrix involves considerable difficulties, and even the solution for semi-matrices of the $X^{-1} A X=D$

I See for example, 'l'urnbull, 9 ; T'urnbull and Aitken, 10 ; Wedderburn, 12 ; Julia, 7 ; Volterra and Hostinsky, 11.

${ }^{2}$ Dienes, 3, 4, 5 .

: Cooke, 1, 2.

4 Wedderburn, 12. 
problem in the Heisenberg-Dirac theory of atom mechanics is only possible under certain conditions. Therefore it is not surprising that at present, owing to the difficulty of establishing the existence of products in infinite matrices, it has been found necessary to restrict in general the class of matrices in this paper to those for which $\sum_{i} 1 / / a_{i i} !$ is convergent.

It will be seen that an examination of the structure necessitates elucidation of certain functional properties, and the incidental properties, e.g. commutability of matrices, have some claim to consideration of their own.

It has been found possible to express infinite lower semi-matrices or matrices which can be transformed into lower semi-matrices (restricted in general to the class $\sum_{i} 1 /\left|a_{i i}\right|$ convergent) in terms of the principal (or unique) idempotent elements ${ }^{1}$. This method of expression enables rational functions of the matrix to be expressed simply in terms of the principal idempotent and nilpotent elements. Throughout this paper capital letters are used to denote infinite lower semi-matrices.

It has been found possible, by algebraic construction of the idempotent and nilpotent elements to extend the class of matrices analysed to certain important special cases. The most important case so dealt with is that in which every element of the principal diagonal is distinct, e.g. The Arithmetic Means.

\section{Preliminary note on the exponential function ${ }^{2}$.}

1.1. Definition. By the "matrix modulus" is meant the matrix obtained by replacing $a_{i i}$ in $A$ by $\left|a_{i k}\right|$, and will be denoted by $|A|$.

Thus the matrix modulus must be distinguished from the Bound as used by Dienes.

Then in the field of lower semi-matrices we have

(i) $|c \cdot A|=|c| \cdot|A|$ where by $A|=| B \mid$ is meant $\left|a_{i k}\right|=\left|b_{i k}\right|$, and $c$ is any scalar matrix, for $\{|c . A|\}_{i k}=c \mid \cdot\{|A|\}_{i k}$.

(ii) $|E|=1$.

(iii) $\{|A+B|\}_{i k}=\left|a_{i k}+b_{i k}\right| \leqq\left|a_{i k}\right|+\left|b_{i k}\right|=\left\{\left|A^{\prime}\right|\right\}_{i k}+\{|B|\}_{i k}$,

3 Cf. Wedderburn, 12.

? Dicnes, 4. 
that is,

that is,

$$
|A+B| \leqq|A|+|B|
$$

(iv) $\{|A \cdot B|\}_{i k}=\left|\sum_{j=k}^{i} a_{i j} b_{j k}\right| \leqq \sum_{j=k}^{i}\left|a_{i j}\right| \cdot\left|b_{j k}\right|=\{|A|\}_{i k} \cdot\{|B|\}_{i k}$,

$$
|A \cdot B| \leqq|A| \cdot \mid B^{\prime} \text {. }
$$

1.2. Theorem. The exponential function exists for every row-finite matrix, all its values are row-finite matrices, and it misses all the nilpotent values in the field.

(i) Since row-finite matrices form an associative field, and in particular are self-associative, the exponential function may be defined as

$$
E(A)=1+\frac{A}{1 !}+\frac{A^{2}}{2 !}+\ldots+\frac{A^{n}}{n !}+\ldots
$$

where the matrix element $\{E(A)\}_{n k}$ is of the form

$$
\{E(A)\}_{n k}=\delta_{n k}+a_{n k}+\frac{\sum_{i} a_{n i} \cdot a_{i k}}{2 !}+\ldots+\frac{\sum_{i} a_{n i} \sum_{i} a_{i j} \ldots \sum_{p} a_{t p} a_{p k}}{n !}+\ldots
$$

Let $b$ be the maximum modulus of the elements in the first $n$ rows of $A$, then

$$
\begin{aligned}
\left|\{E(A)\}_{n k}\right| & \leqq 1+b+\frac{m b^{2}}{2 !}+\ldots+\frac{m^{k} b^{k+1}}{(k+1) !}+\ldots \\
& \leqq e^{m b}
\end{aligned}
$$

where $m$ is the number of elements in the row. 'Therefore $E(A)$ has a definite meaning in the field.

(ii) In the Cauchy product of $E(A) . E(B)$ the term containing the $n$th combined index of $A$ and $B$ is,

$$
\frac{A^{n}}{n !}+\frac{A^{n-1}}{(n-1) !} B+\ldots+\frac{B^{n}}{n !}
$$

which is congruent with $(A+B)^{n} / n$ ! in an associative field when $A B=B A$.

Hence there is absolute equivalence between corresponding powers in $E(A) \cdot E(B)$ and $E(A+B)$.

(iii) Since $E(A+B)=E(A) \cdot E(B)$ when $A B=B A$, put $B=-A$, so that $E(-A)$ is an ordinary element in the field. 
Now from definition $E(0)=1$; hence it follows that

$$
1=E(0)=E(A) \cdot E(-A),
$$

and therefore that it is impossible for $E(A)$ to assume the value zero.

Further suppose that $E(A)=U$ so that $E(n A)=U^{n}$.

Then, since $E(n A)$ cannot be zero, $E(A)$ cannot assume a value $U$ such that $U^{n}=0$, that is $E(A)$ misses all the nilpotent values of the field.

These results can be easily extended to row-bounded, columnfinite, column-bounded, $K$ and $T$ matrices ${ }^{1}$.

2. The Existence Theorem. It becomes necessary to establish the existence of products of the form $\prod_{i}\left(1+\frac{A}{a_{i i}}\right)$.

When $\sum_{i} \mid \frac{1}{a_{i i} \mid}$ is convergent it can be established that

(i) Any product of the form $\prod_{i}\left(1+\frac{A}{a_{i i}}\right)$ exists.

(ii) $\prod_{i=1}^{\infty}\left(1-\frac{A}{a_{i i}}\right)=0$ in the sense that $\lim _{n \rightarrow \infty} \prod_{k=1}^{n}\left(1-\frac{A}{a_{k k}}\right)$

exists and is the zero matrix.

(iii) If $A_{i}=\prod_{k=1}^{\infty}(1)\left(1-\frac{A}{a_{k k}}\right)$ and if $c_{i}=\prod_{i=1}^{\infty}(1)\left(1-\frac{a_{i i}}{a_{k k}}\right)$,

where (i) signifies that the factor(s) where $a_{k k}=a_{i i}$ are omilted, then $A_{i}$ and $c_{i}$ exist, and further $A_{j} A_{k}=0, \quad(j \neq k)$.

2.1. Proof. Using the matrix modulus it is apparent that

$$
1+\left|\frac{A}{a_{i i}}\right|<E\left(\frac{A}{a_{i i}} \mid\right)
$$

ard since $\begin{gathered}A \\ \overrightarrow{a_{i i}}\end{gathered}$ and $\frac{A}{a_{k k}} \mid$ are commutable it follows that

$$
E\left(\left|\frac{A}{a_{i i}}\right|\right) \cdot E\left(\frac{A}{a_{k k}} \mid\right)=E\left(\left|\frac{A}{a_{i i}}\right|+\frac{A}{a_{k k}} \mid\right),
$$

and hence that

$$
\prod_{i}\left(1+\mid \frac{A}{a_{i i}}\right)<E\left(\sum_{i} \mid \frac{A}{a_{i i}}\right)=E\left(|A| \sum_{i} \frac{1}{\left|a_{i i}\right|}\right) .
$$

\footnotetext{
${ }^{1}$ See also Dienes, 3.
} 
By comparison with 1.2 it follows that, since $\sum_{i}\left(1 /\left|a_{i i}\right|\right)$ converges, the right-hand side exists. That is, every such product exists and, in particular, all products of the type $\prod_{i}\left(1-\frac{A}{a_{i i}}\right)$ exist.

2.2. (i) LEMma. Let $U$ be such that the elements in its first $n$ rows are all zero. Let $V$ be commutable with $U$, and such that $v_{n+1, n+1}=0$ for $a$ given $n$. Then in $U V$ the elements in the first $n+1$ rows are all zero. For, if $B=U V$ then

$$
b_{k i}=\sum_{j=1}^{k} u_{k j} v_{j i}
$$

Now $u_{k j}=0$ for $k \leqq n$; hence $b_{k i}=0$ for $k \leqq n$. Further since $B=U V=V U$, it follows that

$$
b_{n+1, i}=\sum_{j=1}^{n+1} v_{n+1, j} u_{j i}
$$

and $u_{j i}=0$ for $j \leqq n$, and $v_{n+1, j}=0$ for $j=n+1$; hence $b_{n+1, i}=0$, that is $b_{k i}=0$ for $k \leqq n+1$.

22 . (ii) In $\left(1-A / a_{11}\right)$ the first row is zero, and in $\left(1-A / a_{22}\right)$ the second element in the principal diagonal is zero; therefore from 2.2. (i) it follows that the elements in the first two rows of

are all zero.

$$
\left(1-A / a_{11}\right)\left(1-A / a_{22}\right)
$$

The complete result then follows by induction, that

is the zero matrix.

$$
\lim _{n \rightarrow \infty} \prod_{i=1}^{n}\left(1-\frac{A}{a_{i i}}\right)
$$

2.3. The existence of $c_{i}$ follows as a direct result of the convergence of $\sum_{i}\left(1 /\left|a_{i i}\right|\right)$ and the existence of $A_{i}$ from 2.1. Further,

$$
A_{j} A_{k}=\prod_{i=1}^{\infty}\left(1-\frac{A}{a_{i i}}\right) \prod_{i=1}^{\infty}\left(1-\frac{A}{a_{i i}}\right),
$$

the rearrangement of the factors given by $a_{i i}=a_{j j}$ being justified by commutability. Hence, by 2.2. (ii), it follows that $A_{j} A_{k}=0,(j \neq k)$.

3. Matrices with Distinct Roots.

Theorem. If in the principal diagonal of $A$ no two elements are equal, then 
(i) $\sum_{i=1}^{\infty} \frac{A_{i}}{c_{i}}=1$, and $\frac{A_{i}}{c_{i}}$ is idempotent.

(ii) $A=\sum_{i=1}^{*} a_{i i} \frac{A_{i}}{c_{i}}$, and further, if $g(x)$ is any function containing only positive and negative powers of $x$, then

$$
g(A)=\sum_{i=1}^{\infty} g\left(a_{i i}\right) \frac{A_{i}}{c_{i}} .
$$

Proof. 3.1. Lemma. If $B$ is commutable with $A$ where $a_{i i} \neq a_{i+k, i+k}$ for all $i$ and $k$, and if the first $n$ elements of the principal diagonal of $B$ are all equal to $c$, then the elements of the first $n$ rows of $B$ other than those of the principal diagonal are zero.

If all the elements of the principal diagonal of $B$ are equal to $c$, then $B=c$.

Using the relation $B A=A B$ it is seen that the second diagonal of $B$ is determined by the equality

$$
b_{i, i-1} a_{i-1, i-1}+c \cdot a_{i, i-1}=c . a_{i, i-1}+a_{i i} b_{i, i-1} .
$$

Therefore $b_{i, i-1}=0,(i \leqq n)$. Hence the third diagonal is governed by

$$
b_{i, i-2} a_{i-2, i-2}+c . a_{i, i-2}=c . a_{i, i-2}+a_{i i} b_{i, i-2,}(i \leqq n),
$$

that is $b_{i, i-2}=0,(i \leqq n)$. Proceeding in this way the $(k+1)$ th diagonal is governed by

$$
\sum_{n=0}^{k} b_{i, i-n} a_{i-n, i-k}=\sum_{n=0}^{k} a_{i, i-n} b_{i-n, i-h}
$$

which, for $i \leqq n$, reduces to

$$
b_{i, i-k} a_{i-k, i-k}=a_{i i} b_{i, i-k}
$$

that is, for $i \leqq n$ the $(k+1)$ th diagonal is zero and the result follows.

3.2. (i) Since $A_{i}==\prod_{k=1}^{\infty}\left(1-\frac{A}{a_{k k}}\right)$, it follows that in the principal diagonal of $A_{i} / c_{i}$ every element is zero except the $i$ th and the $i$ th is 1 . Hence, if $B=\sum_{i=1}^{n} \frac{A_{i}}{c_{i}}$ then $b_{k k}=1$ for $k \leqq n$ and $B$ is commutable with $A$. Then from 3.1 it follows that $b_{k i}=0$ for $k \leqq n$ and $k \neq i$, and hence that $\lim _{n \rightarrow \infty} \sum_{i=1}^{n} \frac{A_{i}}{c_{i}}$ is the unit matrix, and may be expressed $\operatorname{as} \sum_{i=1}^{\infty} \frac{A_{i}}{c_{i}}=1$. 
3.2. (ii) From 2.3 and the above it follows that

$$
\frac{A_{k}}{c_{k}}=\frac{A_{k}}{c_{k}} \sum_{i=1}^{\infty} \frac{A_{i}}{c_{i}}=\left(\frac{A_{k}}{c_{k}}\right)^{2},
$$

that is, $A_{i} / c_{i}$ is idempotent.

3.2. (iii) From 2.2. (ii), since $a_{i i} \neq a_{i+k, i+k}$ for any $i$ or $k$, it follows that $A_{i}\left(1-A / a_{i i}\right)=0$; that is $A\left(A_{i} / c_{i}\right)=a_{i i}\left(A_{i} / c_{i}\right)$ from whence it readily follows that

$$
\lim _{n \rightarrow \infty} A \sum_{i=1}^{n} \frac{A_{i}}{c_{i}}=\lim _{n \rightarrow \infty} \sum_{i=1}^{n} a_{i i} \frac{A_{i}}{c_{i}},
$$

and finally

$$
A=\sum_{i=1}^{\infty} a_{i i} \frac{A_{i}}{c_{i}}
$$

3.2. (iv) Using $A_{j} A_{k}=0$ we have, for $r$ a positive integer,

$$
A^{r}=\sum_{i=1}^{\infty}\left(a_{i i} \frac{A_{i}}{c_{i}}\right)^{r}=\sum_{i=1}^{\infty} a_{i i}^{r} \frac{A_{i}}{c_{i}}
$$

since $A_{i} / c_{i}$ is idempotent.

Further from $A \cdot A^{-1}=1$ it is easily verified that $A^{-1}=\sum_{i=1}^{\infty} a_{i i}^{-1} \frac{A_{i}}{c_{i}}$, and hence that $A^{-r}=\sum_{i=1}^{\infty} a_{i i}^{-r} \frac{A_{i}}{c_{i}}$, and finally that $g(A)=\sum_{i=1}^{\infty} g\left(\dot{a}_{i i}\right) \frac{A_{i}}{c_{i}}$

4. Matrices with Multiple Roots.

Theоnem. Suppose that in the principal diagonal of $A$ there occur equalities between some of the elements, such $a s$, say, $a_{p p}=a_{s 8}=\ldots=a_{v v}$ where the consecutive elements in the sequence of equalities are not necessarily consecutive elements in the diagonal. Then $A$ may be expressed in terms of its principal idempotent and nilpotent elements, with an extension to scalar functions as before.

4.1. Proof. From 3.2 (ii) it is seen that when the roots are distinct $\left(A_{i} / c_{i}\right)\left(1-A_{i} / c_{i}\right)=0$ and the idempotent element may be written as $1-\left(1-A_{i} / c_{i}\right)$ If, however, $a_{i i}=a_{i+1, i+1}$ this is not the case as is shown at once by a consideration of $a_{i+1, i}$. Consideration of $a_{i+1, i}$ suggests, in order to retain the same structural form, the examination of $\left\{\left(A_{i} / c_{i}\right)\left(1-A_{i} / c_{i}\right)\right\}^{2}$ with the possibility of $1-\left(1-A_{i} / c_{i}\right)^{2}$ as the idempotent element. This leads to the following construction. Let 
$m_{i}$ be the "stretch" of the sequence of elements equal with $a_{i i}$ that is, if $a_{s 8}$ is the first and $a_{t t}$ the last of the sequence, then $m_{i}=t+1-s$.

Let $G_{i}=1-\left(1-\frac{A_{i}}{c_{i}}\right)^{m_{i}}$ and consider $U=\sum_{i=1}^{\infty} G_{i}$ where the dash signifies that of the $m_{i}$ congruent terms given by the sequence of elements equal to $a_{i i}$ all but one are omitted. Then by comparison with 3.2 (i) it is seen that $U$ exists.

Further, for values of $k$ such that $a_{k k} \neq a_{i i}$ using $A_{j} A_{k}=0$ it follows that

$$
G_{i} G_{k}=0,
$$

and

that is

$$
U\left(\frac{A_{k}}{c_{k}}\right)^{m_{k}}=\left(\frac{A_{k}}{c_{k}}\right)^{m_{k}} \cdot G_{k},
$$

$$
(U-1)\left(\frac{A_{k}}{c_{k}}\right)^{m_{k}}=-\left(\frac{A_{k}}{c_{k}}\right)^{m_{k}}\left(1-\frac{A_{k}}{c_{k}}\right)^{m_{k}} .
$$

Consider the right-hand side of 4.1 (ii).

Let $B_{1}=\left(A_{k} / c_{k}\right)\left(1-A_{k} / c_{k}\right)$ so that the principal diagonal of $B_{1}$ is zero. Then $B_{1}^{m_{k}}$ has the first $m_{k}$ diagonals zero.

Further, since $A_{j} A_{k}=0$ for values of $j$ such that $a_{j j} \neq a_{k i k}$, it follows that $B_{1 .}^{m_{h}} \sum_{i=1}^{\infty} \frac{A_{i}}{c_{i}}=0$, where the double dash signifies that all terms congruent with, and including, $A_{k} / c_{k}$ are excluded from the summation.

$$
\text { A!so, if } B_{1}^{m_{k}}=B \text { and } \sum_{i=1}^{\infty} \frac{A_{i}}{c_{i}}=C \text { then } B C=C B=0
$$

Now consider the $k=\left(m_{k}+1\right)$ th diagonal of $B$. From (ii) and (iii) this is determined by

$$
\sum_{j=0}^{k} b_{n, n-j} c_{n-j, n-k}=\sum_{j=0}^{k} c_{n, n-j} b_{n-j, n-k}=0 .
$$

That is, since by (i) $b_{n, n-j}=0$ for $j<k$, and $b_{n-j, n-k}=0$ for $j>0$,

$$
b_{n, n-k} c_{n-k, n-k}=c_{n n} b_{n, n-k}=0 \text {. }
$$

In $\sum_{i=1}^{\infty} \frac{A_{i}}{c_{i}}$ the elements of the principal diagonal are non-zero except those which correspond with $a_{k k}$ and the principal diagonal elements of $A$ which are equal to $a_{k k}$. Hence, since the stretch from $c_{n-k, n-k}$ to $c_{n n}$ is greater than $m_{k}$, it follows that $c_{n-k, n-k}$ and $c_{n n}$ cannot both be zero for a fixed $n$. Therefore, using (iv) we have 
$b_{n, n-k}=0$ for all $n$. Similarly $b_{n, n-k-1}=0$ for all $n$, and ultimately it follows that every diagonal of $B$ is zero. Hence 4.1 (ii) becomes

$$
(U-1)\left(\frac{A_{k}}{c_{k}}\right)^{m_{k}}=0
$$

and since this is true for all $k$, we obtain

$$
(U-1) \sum_{k=1}^{\infty}\left(\frac{A_{k}}{c_{k}}\right)^{m_{k}}=0 .
$$

Now in $\sum_{i=1}^{\infty} \frac{A_{i}}{c_{i}}$ every element of the principal diagonal is nonzero, therefore in $\sum_{i=1}^{\infty}\left(\frac{A_{i}}{c_{i}}\right)^{m_{i}}$ every element in the principal diagonal is non-zero, and it immediately follows that $u_{n n}$ is 1 for all $n$, and, by successive determinations of the diagonals, that $u_{n k}=0$ for $k \neq n$; that is, $U$ is the unit matrix.

Using 4.1 (i) and 4.1 (iii), it follows from the definition of $U$ and $G_{i}$ that

4.1. (iv)

that is, $G_{k}$ is idempotent.

$$
G_{k} U=\left(G_{k}\right)^{2}
$$

Let

$$
\eta_{k}=\left(1-\frac{A}{a_{k k}}\right) \cdot G_{k}
$$

so that

$$
\eta_{k}^{s_{k}}=\left(1-\frac{A}{a_{k k}}\right)^{\varepsilon_{k}} \cdot G_{k},
$$

since $G_{k}$ is idempotent.

Let $s_{k}$ be the actual number of elements in the total sequence $a_{k k}=r_{j}=\ldots=a_{m m}$. Then

$$
\eta_{k}^{g_{k}}=f(A) \prod_{i=1}^{\infty}\left(1-\frac{A}{a_{i i}}\right),
$$

that is, $\eta_{k}$ is nilpotent with index $s_{k}$. Thus we have

$$
A=A U=A \sum_{i=1}^{\infty} G_{i}=\sum_{i=1}^{\infty} a_{i i}\left(G_{i}-\eta_{i}\right)
$$

where $G_{i}$ is idempotent and $\eta_{i}$ is nilpotent with index $s_{i}$.

Further, it is readily seen that if $r$ is a positive integer

$$
A^{r}=\sum_{i=1}^{\infty} a_{i i}^{r}\left(G_{i}-\eta_{i}\right)^{r}=\sum_{i=1}^{\infty} a_{i i}^{r}\left\{G_{i}+\left(1-\eta_{i}\right)^{r}-1\right\}
$$

that is, if $g(x)$ is a scalar polynomial in $x$, then 
4.1. (vii) $\quad g(A)=\sum_{i=1}^{\infty}\left\{g\left(a_{i i}\right) G_{i}+a_{1} \eta_{i}+a_{2} \eta_{i}^{2}+\ldots+a_{n} \eta_{i}^{n}\right\}$,

where $n$ is the degree of the polynomial, and $a_{y}$ is derived from powers of $a_{i i}$.

\section{UNIQUENESS.}

The matrices $G_{i}$ are uniquely determined. For, let $K_{i}(i=1,2, \ldots)$ be any matrices such that

(i) $A K_{i}=K_{i} A$,

(ii) $\left(1-A / a_{i i}\right) \cdot K_{i}$ is nilpotent,

(iii) $\sum_{i=1}^{\infty} K_{i}=1, K_{i}$ is idempotent and non-zero.

Then $K_{i}=G_{i},(i=1,2, \ldots)$.

For, if $\psi_{j}=\left(1-A_{i} / a_{j j}\right) \cdot K_{j}$, we have, since $K_{i}$ and $G_{i}$ are commutable with each other and with $A$, that

and

$$
a_{j j} G_{i} \psi_{j}=\left(a_{j j}-A\right) \cdot K_{j} G_{i}
$$

that is

$$
a_{i i} K_{j} \eta_{i}=\left(a_{i i}-A\right) \cdot K_{j} G_{i},
$$

$$
a_{j j} G_{i} \psi_{j}-a_{i i} K_{j} \eta_{i}=\left(a_{j j}-a_{i i}\right) K_{j} G_{i}
$$

But if $a$ is the higher index of $\psi_{j}$ and $\eta_{i}$, and if both sides are raised to the power $2 \alpha$, then since all the matrices are commutable, and since $K_{j} G_{i}$ is idempotent, the left-hand side becomes zero and the right-hand side does not, unless $a_{j j}=a_{i i}$.

Hence $K_{j} G_{i}=0$ when $a_{i i} \neq a_{j j}$.

Therefore from (iii) we have

$$
K_{j}=K_{j} \sum_{i=1}^{\infty} G_{i}=K_{j} G_{j}=G_{j} \sum_{j=1}^{\infty} K_{j}=G_{j}
$$

which proves the uniqueness of the $G_{i}$. Hence they may be called the "Principal" jdempotent elements.

An algebraic construction for particular cases.

6. Case 1. Suppose that $A$ is any lower semi-matrix such that every element of the principal diagonal is distinct, that is $a_{i i} \neq a_{k k}$ for any $k, k \neq i$.

Let $B_{k}, k$ being a fixed integer, be such that

(i) $B_{k} A=A B_{k}$

(ii) $\left(B_{k}\right)_{i i}=0$ for $i \neq k$

$$
=1 \text { for } i=k \text {. }
$$


Then $(a) \sum_{i=1}^{\infty} B_{i}=1$,

(b) $B_{i}$ is idempotent for all $i$,

(c) $A=\sum_{i=1}^{\infty} a_{i i} B_{i}$.

6.1. Proof. Determining $B_{k}$ diagonally the element $\left(B_{k}\right)_{n m}$ is given, using (i), by

$$
\sum_{j=m}^{n}\left(B_{k}\right)_{n j} a_{j m}=\sum_{j=m}^{n} a_{n j}\left(B_{k}\right)_{j m} .
$$

(a) $m=n$ is determined by $\left(B_{k}\right)_{n n} a_{n n}=a_{n n}\left(B_{k}\right)_{n n}$, that is $\left(B_{k}\right)_{n n}$ may be fixed to satisfy (ii).

(b) $m=n-1$. Having fixed $\left(B_{k}\right)_{n n}$ the element $\left(B_{k}\right)_{n, n-1}$ is determined by

$$
\left(B_{k}\right)_{n, n-1} a_{n-1, n-1}+\left(B_{k}\right)_{n n} a_{n, n-1}=a_{n, n-1}\left(B_{k}\right)_{n-1, n-1}+a_{n n}\left(B_{k}\right)_{n, n-1} \text {, }
$$

that is since $a_{n n} \neq a_{n-1, n-1}$ the element $\left(B_{k}\right)_{n, n-1}$ is fixed.

(c) Proceeding in this way the matrix $B_{k}$ is uniquely determined.

\subsection{The form of $B_{k}$.}

By inspection of $B_{k}$ by means of the condition (i) it is at once apparent that $B_{k}$ has the simple rectangular form,

$$
\begin{aligned}
\left(B_{k}\right)_{n j} & =0 \text { for } n<k, \quad(j=1,2, \ldots n), \\
& =0 \text { for } n>k, \quad(j=k+1, k+2, \ldots n) .
\end{aligned}
$$

6.3. $B_{k} B_{j}=0$ for $k \neq j$.

Let $B_{k} B_{j}=C$ then $C A=A C$, and it follows that

(a) every element of the principal diagonal of $C$ is zero,

(b) a diagonal determination of $C$ gives

$$
\sum_{j=m}^{n} c_{n j} a_{j m}=\sum_{j=m}^{n} a_{n j} c_{j m},
$$

whence it follows, using $(a)$ and condition (i) that every element of $C$ is zero; that is $B_{k} B_{j}=0$.

6.4. $B_{k}$ is idempotent for all $k$.

Let $B_{k}\left(1-B_{k}\right)=C$. Then as in 6.3 it follows that $C$ is the zero matrix, that is $B_{k}=B_{k}^{2}$. 
6.5. From 6.3 and 6.4 it follows that $B_{k}\left(1-\sum_{i=1}^{\infty} B_{i}\right)=0$ is true for all $k$.

(a) The principal diagonal of $\left(1-\sum_{i=1}^{\infty} B_{i}\right)$ is zero.

(b) The second diagonal of this matrix is determined by

$$
\left(B_{k}\right)_{n n}\left(1-\sum_{i=1}^{\infty} B_{i}\right)_{n, n-1}=0 \text {. }
$$

Letting $k=n$ and therefore $\left(B_{k}\right)_{n n}=1$ it follows that

$$
\left(1-\sum_{i=1}^{\infty} B_{i}\right)_{n, n-1}=0 \text {, }
$$

that is, the second diagonal is zero.

(c) Proceeding in this way it is established that

$$
1-\sum_{i=1}^{\infty} B_{i}=0
$$

that is,

$$
\sum_{i=1}^{\infty} B_{i}=1
$$

6.6. Next consider the matrix $B_{k}\left(a_{k k}-A\right) \equiv C$ say.

Then as in 6.3 it follows that $C$ is the zero matrix, that is $A B_{k}=a_{k k} B_{k}$ is true for all $k$. Hence using 6.5,

where $B_{i}$ is idempotent.

$$
A=\sum_{i=1}^{\infty} a_{i i} B_{i}
$$

6.7. As an example of this case may be taken the very important Arithmetic means. If those of the first order are taken it may be observed that in this case $B_{1}$ is a first column matrix where every element is 1 .

7. Case 2. Suppose that in the principal diagonal of $A$ there occur sets of consecutive equalities such as

$$
a_{k k}=a_{k+1, k+1}=\ldots=a_{k+m, k+m}
$$

and $a_{i i} \neq a_{k k}$ for any value of $i$ outside the set $k, \ldots k+m$.

7.1. With each set associate one (and only one) lower semi-matrix $B_{k}$ such that

(i) $B_{k} A=A B_{k}$.

Then $B_{k}$ may be determined such that

(ii) $\left(B_{k}\right)_{i i}=1$ for $i=k, k+1, \ldots k+n$, $=0$ elsewhere. 
It readily follows from inspection that we may put

(iii) $\left(B_{k}\right)_{i j}=0$ for $i<k$ and all $j$,

(iv) $\left(B_{k}\right)_{i j}=0$ for $j>k+m$ and all $i$,

(v) $\left(B_{k}\right)_{i j}=0$ for $k<i \leqq k+m, j \geqq k$,

and that $B_{k}$ is then uniquely determined.

7.2. Let $B_{k}\left(1-B_{k}\right)=C$.

Then $C A=A C, c_{i i}=0$ for all $i$, and $c_{n j}=0$ when $a_{n n}=a_{j j}$.

7.2. (i) In the diagonal determination of $C$ it then follows that the second diagonal is given by

$$
c_{n, n-1} a_{n-1, n-1}=a_{n n} c_{n, n-1}
$$

and hence it follows that $c_{n, n-1}=0$ for all $n$.

7.2. (ii) Proceeding in this way it becomes established that $C$ is the zero matrix; that is $B_{k}=B_{k}^{2}$ for all $k$.

7.3. In the same way it follows that $B_{k} B_{j}=0$ for $k \neq j$.

7.4. Thus

$$
B_{k}\left(1-\sum_{i=1}^{\infty} B_{i}\right)=0
$$

is true for all $k$, and hence as in 6.5 it follows that

$$
\sum_{i=1}^{\infty} B_{i}=1
$$

7.5. Now let $B_{k}\left(\iota_{k k}-A\right)=-\eta_{k}$.

Then since the principal diagonal of $\eta_{k}$ is zero it follows that in $\left(\eta_{k}\right)^{m+1}$ the first $m+1$ diagonals are zero. Therefore it follows that when $a_{n n}=a_{j j}$ then $a_{n j}=0$. Hence, as in previous arguments, it follows that $\left(\eta_{k}\right)^{m+1}$ is the zero matrix, that is, $\eta_{k}$ is nilpotent with index $\leqq m+1$, and finally that $A B_{k}=\left(a_{k k} B_{k}+\eta_{k}\right)$ is true for all $k$, and that, using 7.4

$$
A=\sum_{i=1}^{\infty}\left(a_{i i} B_{i}+\eta_{i}\right)
$$

8.1. The extension to the case where $m \rightarrow \infty$ introduces no contradiction or difficulty, but does introduce the conception of a nilpotent matrix of infinite index. Such a matrix, though dealt with adequately by the extended argument, is not unique, as any lower semi-matrix with every element of the principal diagonal zero is nilpotent with infinite index. 
A special case of the extended argument is the matrix in which every element of the principal diagonal is equal to $c$. In this matrix the idempotent element is the principal diagonal divided by $c$ (that is, the unit matrix) and the rest of the matrix is the nilpotent element.

8.2. The extension to the general case where there is no restriction on the existence of equalities in the principal diagonal seems too unwieldy for algebraic argument.

\section{BIBLIOGRAPHY.}

1. R. G. Cooke, Some solutions of the matrix equation $A X-X A=1$. Journal Londun Math. Soc., 8 (1933), 107-109.

2. R. G. Cooke, The transformation of some classes of in finite matrices into diagonal matrices. Journal London Math. Soc., 8 (1933), 167-175.

3. P. Dienes, The Taylor series (Oxford, 1931).

4. P. Dienes, The exponential function in linear algebras. Quarterly Journal (Oxford Series), 1 (1930), 300-309.

5. P. Dienes, Notes on Linear Equations in infinite matrices. Quarterly Journal (Oxford Series), 3 (1932), 253-268.

6. H. Hilton, Linear substitutions (Oxford, 1914).

7. G. Julia, Intruduction mathérnctique aux théories quantiques (Paris, 1936).

8. F. Riesz, Les systemes d'equations linéaires a une infinité d'inconnues (Paris, 1913).

9. H. W. Turnbull, The theory of determinants, matrices and intariants (Blackie, 1928).

10. H. W. Turnbull and A. C. Aitken, An introduction to the theory of canonical matrices (Blackie, 1932).

11. V. Volterra and B. Hostinsky, Operations infinitesimales linéaires (Paris, 1938).

12. J. H. M. Wedderburn, Lectures on matrices (Amer. Math. Soc., New York, 1934).

\section{Birkbeck College,}

LoNDON, E.C. 4. 